\title{
Qualidade de vida de estudantes de graduação e pós-graduação em Odontologia
}

\author{
Quality of life of students of undergraduate and graduate in Dentistry \\ Calidad de vida de estudiantes de pregrado y postgrado de Odontología \\ João Rafael AMADEU ${ }^{1}$ \\ Mirella Martins JUSTI ${ }^{2}$ \\ ${ }^{1}$ UNESP Universidade Estadual Paulista, 16015-050 Araçatuba-SP, Brasil \\ ${ }^{2}$ UNIP Universidade Paulista, 16018-555 Araçatuba - SP, Brasil
}

\begin{abstract}
Resumo
Segundo a Organização Mundial de Saúde (OMS) a qualidade de vida (QV) pode ser definida como "a percepção do indivíduo de sua posição na vida, no contexto da cultura e sistema de valores nos quais ele vive e em relação aos seus objetivos, expectativas, padrões e preocupações”. Para se mensurar a QV um dos instrumentos utilizados é o questionário WHOQOLbref, que foi desenvolvido pelo Grupo de Qualidade de Vida da Organização Mundial de Saúde para se avaliar este aspecto de forma rápida e objetiva, sendo ele a versão abreviada do WHOQOL-100. O instrumento consta de 26 questões que abrangem diferentes facetas dentro de quatro domínios diferentes, que são o físico, o psicológico, das relações sociais e o ambiente. O objetivo deste estudo foi a aplicação do WHOQOL-Bref em uma amostra de 150 estudantes de graduação e pós-graduação de uma Faculdade de Odontologia de uma universidade pública do Estado de São Paulo com a finalidade de avaliar a qualidade de vida dos estudantes em vários quesitos socioeconômicos. A média geral da qualidade de vida dos estudantes foi de 62,4 . O domínio com melhor avaliação foi o das Relações Sociais $(69,3)$, seguido pelo Psicológico $(64,3)$ e Ambiente $(64,0)$, sendo o Domínio Físico $(51,7)$ o pior avaliado. Por meio deste estudo foi possível a identificação dos pontos pertinentes da qualidade de vida dos estudantes de Odontologia, percebendo muitas das dificuldades vivenciadas no dia a dia e que afetam diretamente este aspecto, além de também buscar traçar algumas estratégias que contribuam para sua melhoria.
\end{abstract} Descritores: Qualidade de Vida; Odontologia; Educação Superior.

\begin{abstract}
Accordingly to World Health Organization (WHO), quality of life (QV) can be defined as "an individual's perception of his position in life in the context of the culture and value systems in which he lives and in relation to his goals, expectations, standards and concerns". For measuring QV, the WHOQOL-bref is a questionnaire developed by World Health Organization Quality of Life Group in order to evaluate the quality of life in a quick and objective way; this test is the abbreviated version of WHOQOL-100. The instrument involves 26 questions covering different facets within four different domains, which are physical, psychological, social relations and environment. The objective of this study was the application of the WHOQOLBref in a sample of 150 undergraduate and graduate students of a Faculty of Dentistry at a public university in the State of São Paulo with the purpose of evaluating quality of life of students in various socioeconomic aspects. The general average of the quality of life of students was 62.4. The domains with the best evaluation were Social Relations (69.3), followed by Psychological (64.3) and Environment (64.0), the Physical Domain (51.7) was the worst evaluated aspect. Through this study was possible to identify relevant points about the quality of life of students Dentistry, perceiving many of the difficulties they experienced and affect their quality of life, as well as seeking strategies for contributions to the improvement of quality of life. Descriptors: Quality of Life; Dentistry; Education, Higher.
\end{abstract}

\section{Resumen}

Según la Organización Mundial de la Salud (OMS), la calidad de vida (QV) puede definirse como "la percepción del individuo de su posición en la vida, en el contexto de la cultura y el sistema de valores en los que vive y en relación con sus objetivos, expectativas, patrones y preocupaciones". Para medir la QV uno de los instrumentos utilizados es el cuestionario WHOQOLbref, que fue desarrollado por el Grupo de Calidad de Vida de la Organización Mundial de la Salud para evaluar este aspecto de forma rápida y objetiva, siendo la versión abreviada del WHOQOL-100. El instrumento consta de 26 cuestiones que abarcan diferentes facetas dentro de cuatro dominios diferentes, que son el físico, el psicológico, las relaciones sociales y el ambiente. El objetivo de este estudio fue la aplicación del WHOQOL-Bref en una muestra de 150 estudiantes de graduación y posgrado de una Facultad de Odontología de una universidad pública del Estado de São Paulo con la finalidad de evaluar la calidad de vida de los estudiantes en varios los aspectos socioeconómicos. El promedio general de la calidad de vida de los estudiantes fue de 62,4. El dominio con mejor evaluación fue el de las Relaciones Sociales $(69,3)$, seguido por el Psicológico (64,3) y Ambiente $(64,0)$, siendo el Dominio Físico $(51,7)$ el peor evaluado. Por medio de este estudio fue posible la identificación de los puntos pertinentes de la calidad de vida de los estudiantes de Odontología, percibiendo muchas de las dificultades vivenciadas en el día a día y que afectan directamente este aspecto, además de buscar trazar algunas estrategias que contribuyan a su mejoría.

Descriptores: Calidad de Vida; Odontología; Educación Superior.

\section{INTRODUÇÃO}

Embora notoriamente o interesse do tema Qualidade de Vida (QV) tenha aumentado significativamente nas últimas décadas, sua definição ainda é algo vago e subjetivo, pois envolve contextos individuais em quesitos como a saúde, bens matérias e bem estar ${ }^{1}$.

Uma das primeiras aparições da expressão "Qualidade de Vida" ocorreu graças ao ex-presidente norteamericano Lyndon Johnson, que afirmou nos anos 60 que uma nação não pode medir seus objetivos de vida apenas através do balanço dos bancos, mas também através da QV que proporciona às pessoas ${ }^{2}$. Já nas décadas seguintes, a expressão QV passou a ser utilizada para estudos de nível de vida, critérios econômicos-sociais e também em discursos genéricos na mídia, sendo amplamente utilizado para criar condições que somem valor e qualidade à vida das pessoas ${ }^{3}$.

Apesar de ser um conceito regularmente empregado no cotidiano, quando recorremos à literatura há uma série de definições para QV que narram sobre fatores subjetivos da experiência humana como, por exemplo, Wilheim ${ }^{4}$ que define a QV com uma sensação de bem-estar individual. Já para Limongi França ${ }^{5}$ a QV seria uma percepção do bem estar, a partir das necessidades individuais, expectativas de vida e ambiente social e econômico. 
Para o Grupo de Qualidade de Vida da Organização Mundial da Saúde $^{6}$ a definição de QV é "a percepção do indivíduo de sua posição na vida, no contexto da cultura e sistema de valores nos quais ele vive e em relação aos seus objetivos, expectativas, padrões e preocupações".

Juntamente com a evolução do conceito de QV, também houve um crescimento pelo interesse de mensurá-la, bem como seus níveis. Sendo assim, diversos instrumentos de avaliação foram desenvolvidos, em sua maior parte produzidos nos Estados Unidos, e posteriormente traduzidos e adaptados para outras culturas.

Um dos instrumentos desenvolvidos para que se pudesse avaliar e ponderar de forma científica a qualidade de vida de um indivíduo foi o WHOQOL-100, desenvolvido pela Divisão de Saúde Mental da Organização Mundial da Saúde (OMS). Essa ferramenta se baseia em um questionário com 100 questões, que são referentes a seis domínios, sendo eles: físico, psicológico, nível de independência, relações sociais, meio ambiente e espiritualidades. Essas cem questões apresentadas no formulário estão divididas em 24 facetas, sendo que cada uma conta com 4 questões. Para complementar o teste, há ainda uma $25^{\mathrm{a}}$ faceta que é composta de perguntas gerais sobre a qualidade de vida ${ }^{7}$. O WHOQOL-100 é um método validado em diversos países, com diversas publicações no meio científico ${ }^{8}$, sendo que no Brasil sua tradução e aplicação foi detalhada por Fleck et al. ${ }^{9}, 1999$.

Para facilitar sua aplicação e procurando ajustar a necessidade de testes mais rápidos, foi desenvolvido o WHOQOL-bref ${ }^{10}$, que é composto por 26 questões, sendo duas questões sobre a autoavaliação da QV e 24 questões representando cada uma das facetas do WHOQOL-100. Para a composição das questões do WHOQOL-bref, foi selecionada a questão de cada faceta que apresentava a maior correlação com o escore médio de todas as facetas ${ }^{10}$. A versão brasileira do teste WHOQOL-bref também foi traduzida e ajustada por Fleck et al. ${ }^{9}$.

Pesquisas utilizando o WHOQOL-bref e outros parâmetro para medir a qualidade de vida já são realizadas há mais de 30 anos. Porém para os graduandos em Odontologia é notória a falta de estudos e sabendo que a população universitária no Brasil está aumentando a cada ano, o monitoramento de informações importantes e de fatores demográficos e socioambientais, que possam estar relacionadas com a saúde e desempenho acadêmico é fundamental para a formulação de políticas governamentais e programas de intervenção ${ }^{11}$.

Assim, o presente estudo teve por objetivo avaliar a qualidade de vida de graduandos e pós-graduandos de uma faculdade de Odontologia de uma universidade pública do Estado de São Paulo, a fim de contribuir para a análise de qualidade de vida, e assim melhor compreensão da mesma na pós-modernidade, além da produção de dados que possam ser utilizados para a obtenção de uma melhor satisfação dos alunos em sua formação.

\section{MATERIAL E MÉTODO}

A pesquisa intitulada "Qualidade de Vida de Estudantes de Graduação e Pós-Graduação em Odontologia" trata-se de um estudo descritivo e transversal realizado com os alunos de uma faculdade de Odontologia de uma universidade pública do Estado de São Paulo. Para se avaliar a qualidade de vida dos estudantes foi utilizado o WHOQOL-bref, que é um formulário com 26 questões (Tabela 1), desenvolvido pela WHO, e validado em português $^{12}$, sendo que duas questões são de caráter geral sobre a qualidade de vida e as outras 24 visam ponderá-la em quatro domínios, sendo eles: físico, psicológico, relações sociais e ambiente.

Tabela 1. Questões, domínios e facetas do WHOQOL-bref

\begin{tabular}{l|c}
\multicolumn{1}{c|}{ Questão } & Faceta \\
\hline $\begin{array}{l}\text { 1 - Como você avaliaria sua } \\
\text { qualidade de vida? } \\
\mathbf{2} \text { - Quão satisfeito(a) você está com } \\
\text { a sua saúde? }\end{array}$ & Qualidade de vida \\
\multicolumn{2}{c}{ Domínio Físico } \\
\hline \multicolumn{3}{c}{ Questão } \\
\hline $\begin{array}{l}\text { 3 - Em que medida você acha que } \\
\text { sua dor (física) impede você de fazer }\end{array}$ & Dor e desconforto \\
\end{tabular}

o que você precisa?

4 - O quanto você precisa de algum tratamento médico para levar sua vida diária?

10 - Você tem energia suficiente para seu dia a dia?

15 - Quão bem você é capaz de se locomover?

16 - Quão satisfeito(a) você está com o seu sono?

17 - Quão satisfeito(a) você está

com sua capacidade de

desempenhar as atividades do seu dia a dia?

18 - Quão satisfeito(a) você está

com sua capacidade para o trabalho?

\begin{tabular}{c} 
Domínio Psicológico \\
\hline Questão \\
\hline
\end{tabular}

5 - O quanto você aproveita a vida?

Pensar, aprender, memória e concentração

6 - Em que medida você acha que a sua vida tem sentido?

7 - O quanto você consegue se concentrar?

11 - Você é capaz de aceitar sua aparência física?

19 - Quão satisfeito(a) você está consigo mesmo?

Energia e fadiga

Sono e repouso

Mobilidade

Dependência de medicação ou de tratamentos

Atividades da vida cotidiana

26 - Com que frequência você tem sentimentos negativos tais como mau humor, desespero, ansiedade, depressão?

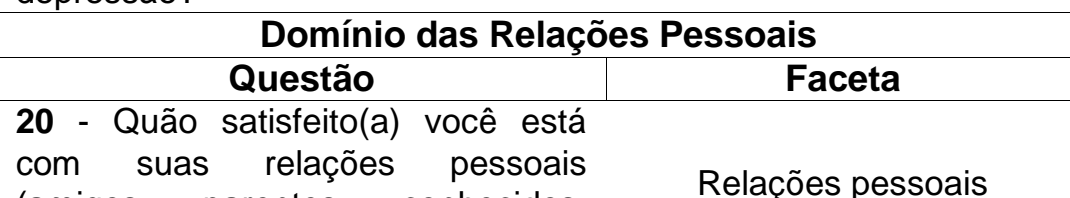

com suas relaços pessoais (amigos, parentes, conhecidos, colegas)?

21 - Quão satisfeito(a) você está com sua vida sexual?

22 - Quão satisfeito(a) você está com o apoio que recebe de seus amigos?

\begin{tabular}{|c|c|}
\hline \multicolumn{2}{|c|}{ Domínio Meio Ambiente } \\
\hline Questão & Faceta \\
\hline $\begin{array}{l}8 \text { - Quão seguro(a) você se ser } \\
\text { em sua vida diária? }\end{array}$ & Seg \\
\hline $\begin{array}{l}9 \text { - Quão saudável é o seu ambi } \\
\text { físico (clima, barulho, polui }\end{array}$ & (poluiçã \\
\hline
\end{tabular}

físico (clima, barulho, poluição, atrativos)?

12 - Você tem dinheiro suficiente para satisfazer suas necessidades? 13 - Quão disponíveis para vocé estão as informações que precisa no seu dia a dia?

14 - Em que medida você tem oportunidades de atividade de lazer? 23 - Quão satisfeito(a) você está com as condições do local onde mora?

24 - Quão satisfeito(a) você está com o seu acesso aos serviços de saúde?

25 - Quão satisfeito(a) você está com o seu meio de transporte?
Sentimentos positivos

Pensar, aprender, memória e concentração Imagem corporal e aparência

Autoestima

Sentimentos negativos

Atividade sexual

Suporte (apoio) socia

Recursos financeiros

Oportunidades de adquirir novas informações e habilidades Participação em, e oportunidades de recreação/lazer

Ambiente no lar

Cuidados de saúde e sociais: disponibilidade e qualidade

Transporte 
A instituição pesquisada contava em julho de 2017 com 568 alunos regularmente matriculados na graduação e 177 na pós-graduação. Com o propósito de assegurar uma representatividade e ter um nível de significância foram entrevistados uma porcentagem superior a $15 \%$ dos alunos perfazendo total de 150 respondentes, sendo que 120 cursavam graduação e 30 pós-graduação.

Para que o estudante fosse elegível para o estudo era necessário que este fosse maior de idade, estivesse regularmente matriculado, seja na graduação ou na pósgraduação, e que estivesse de acordo com as condições apresentadas no Termo de Consentimento Livre e Esclarecido (TCLE), as quais estão de acordo com os padrões pré-estabelecidos pelo Comitê de Ética. Foram entrevistados estudantes de ambos os gêneros e de todos os semestres da graduação e pós-graduação.

Como o preenchimento do WHOQOL-bref foi de caráter voluntário e entrevistado possuía total liberdade para solicitar sua exclusão da pesquisa a qualquer momento, sem ônus ou qualquer prejuízo. Assim, foram excluídos da pesquisa os alunos que não se sentiram motivados a participar e também aqueles que começaram a responder ao formulário e desistiram ou responderam menos de 21 questões.

Algumas informações complementares foram acrescentadas ao questionário, como uma avaliação socioeconômica, onde se investigou gênero, idade, situação laboral (se o estudante apenas estudava ou se também trabalhava), com quem morava, se praticava algum tipo de atividade física e a renda familiar.

Os dados foram organizados e processados através do programa estatístico IBM Statistical Package for the Social Sciences (SPSS), versão 23.0. As variáveis foram analisadas conforme modelo estatístico proposto pelo WHOQOL GROUP - Escore Bruto (EB) e os Escores Transformados 4-20 (ET4-20) e 0-100 (ET0-100)

Aplicou-se o teste Mann-Whitney para comparar os escores de dois grupos independentes e o teste de KruskalWallis para comparar os escores de dois ou mais grupos independentes, utilizando-se $\mathrm{p}<0,05$ como valor de referência para a significância estatística.

O projeto foi enviado ao Comitê de Ética em Pesquisa da Universidade Paulista - UNIP - Vice-Reitoria de Pesquisa e Pós-Graduação, através da Plataforma Brasil, conforme Certificado de Apresentação para Apreciação Ética (CAAE) 70083217.4.0000.5512, respeitando-se todos os aspectos éticos dos estudos envolvendo seres humanos.

\section{RESULTADOS}

Foram avaliados 150 estudantes, sendo que desse total, 82 eram graduandos no período integral $(54,7 \%), 46$ eram graduandos do período noturno $(30,7 \%)$ e 22 eram pós-graduandos, de programas de mestrado e doutorado. $(14,6 \%)$ (Tabela 2).

A maior parte dos estudantes se identificou como do gênero feminino (61,3\%), já os que se identificam com o gênero masculino representam $38,7 \%$ dos entrevistados. A faixa etária mais representativa foi entre 21-22 anos $(36,0 \%)$, seguida por $23-24$ anos $(34,7 \%)$, mais de 25 anos $(16,7 \%)$ e $18-20$ anos $(12,6 \%)$.

Sobre a situação laboral, $78,7 \%$ dos entrevistados responderam que apenas estudam, $14,0 \%$ estudam e trabalham informalmente e 7,3\% estudam e trabalham formalmente. Dentre dos que responderam o questionário, $58,0 \%$ informaram que moravam com amigos, $17,3 \%$ sozinhos, $14,7 \%$ com os pais, $6,7 \%$ familiares e $3,3 \%$ residiam com companheiro ou companheira. 61,3\% dos respondentes relataram praticar atividade física contra $38,7 \%$ que não praticavam.

A média simples da qualidade de vida dos estudantes após a aplicação do instrumento WHOQOL-bref e analisado os seus dados foi de 62,4 com um desvio padrão de $\pm 13,1$. A primeira pergunta do questionário indagou aos entrevistados como eles autoavaliariam sua qualidade de vida, $42,7 \%$ respondeu que avalia como boa; $29,8 \%$ como regular; $20,2 \%$ como ótima, $6,5 \%$ como ruim e $0,8 \%$ como péssima.

A segunda questão era uma autoavaliação sobre quão satisfeito o estudante estaria com sua saúde, 39,0\% responderam estar satisfeitos; $31,7 \%$ não satisfeitos nem insatisfeitos, 14,6\% informaram estar muito satisfeitos; $13,8 \%$ estar insatisfeito e não houve resposta para a opção "muito insatisfeito".

\begin{tabular}{lc} 
Tabela 2. Características gerais da amostra & \\
\hline Idade & $\mathbf{N}$ \\
$18-20$ anos & $19(12,6 \%)$ \\
$21-22$ anos & $54(36,0 \%)$ \\
$23-24$ anos & $52(34,7 \%)$ \\
Mais de 25 anos & $25(16,7 \%)$ \\
\hline Gênero & \\
\hline Feminino & $92(61,3 \%)$ \\
Masculino & $58(38,7 \%)$ \\
\hline Período & \\
Integral & $82(54,7 \%)$ \\
Noturno & $46(30,7 \%)$ \\
Pós-graduação & $22(14,6 \%)$ \\
\hline Situação laboral & \\
Apenas estudam & $118(78,7 \%)$ \\
Estudam e trabalham & $21(14,0 \%)$ \\
informalmente & \\
Estudam e trabalham & $11(7,3 \%)$ \\
formalmente & \\
\hline Com quem mora & \\
Sozinho(a) & $26(17,3 \%)$ \\
Pais & $22(14,7 \%)$ \\
Familiares & $10(6,7 \%)$ \\
Amigos(as) & $87(58,0 \%)$ \\
Companheiro(a) & $5(3,3 \%)$ \\
\hline Renda Familiar & \\
Até R $\$ 1.874,00$ & $18(12,0 \%)$ \\
R $\$ 1.874,01$ a R $\$ 3.748,00$ & $41(27,3 \%)$ \\
R $\$ .748,01$ a R $\$ 9.370,00$ & $23(15,3 \%)$ \\
R 9.370,01 a R $18.740,00$ & $58(38,7 \%)$ \\
R $\$ 18.740,01$ ou mais & \\
\hline Atividade Física & \\
Praticante & \\
Não praticante & \\
\hline & \\
\hline
\end{tabular}

As outras 24 questões eram sobre quatro domínios diferentes (físico, psicológico, relações sociais e ambiente) e para uma melhor visualização dos dados segue na Tabela 3 os valores médios dos escores destes domínios, com desvio padrão (DP), seguido dos valores máximos e mínimos de cada um.

Tabela 3 - Escores máximo e mínimo, escore médio e desvio padrão da QV dos estudantes

\begin{tabular}{lccc}
\multicolumn{1}{c}{ Domínios } & Média \pm DP & Máximo & Mínimo \\
$\begin{array}{l}\text { Domínio I } \\
\text { Físico }\end{array}$ & $51,7 \pm 12,9$ & 81,7 & 17,8 \\
$\begin{array}{l}\text { Domínio II } \\
\text { Psicológico }\end{array}$ & $64,3 \pm 12,2$ & 91,7 & 33,3 \\
$\begin{array}{l}\text { Domínio III } \\
\text { Relações Sociais }\end{array}$ & $69,3 \pm 21,2$ & 100,0 & 0,0 \\
$\begin{array}{l}\text { Domínio IV } \\
\text { Ambiente }\end{array}$ & $64,0 \pm 17,9$ & 100,0 & 12,5 \\
\hline
\end{tabular}


A Tabela 4 apresenta a estratificação da Qualidade de Vida das variáveis socioeconômicas e variáveis do estilo de vida.

Tabela 4. Estratificação da Qualidade de Vida das variáveis socioeconômicas e variáveis do estilo de vida

\begin{tabular}{|c|c|c|}
\hline Variável & Média \pm DP & p-valor \\
\hline Atualmente cursa & & 0,582 \\
\hline Graduação Integral & $66,9 \pm 14,3$ & \\
\hline Graduação Noturno & $63,9 \pm 16,1$ & \\
\hline Pós-graduação & $67,2 \pm 16,6$ & \\
\hline Gênero & & 0,070 \\
\hline Masculino & $60,7 \pm 12,7$ & \\
\hline Feminino & $65,1 \pm 13,5$ & \\
\hline Idade & & 0,268 \\
\hline 18-20 anos & $67,5 \pm 11,6$ & \\
\hline 21-22 anos & $61,0 \pm 13,5$ & \\
\hline 23-24 anos & $61,4 \pm 13,6$ & \\
\hline Mais de 25 anos & $63,4 \pm 12,3$ & \\
\hline Situação Laboral & & 0,904 \\
\hline Apenas estuda & $62,4 \pm 13,9$ & \\
\hline $\begin{array}{l}\text { Estuda e trabalha } \\
\text { formalmente }\end{array}$ & $62,4 \pm 9,56$ & \\
\hline $\begin{array}{l}\text { Estuda e trabalha } \\
\text { informalmente }\end{array}$ & $61,1 \pm 10,0$ & \\
\hline Com quem mora & & 0,559 \\
\hline Sozinho(a) & $61,6 \pm 12,2$ & \\
\hline Pais & $59,5 \pm 15,2$ & \\
\hline Familiares & $68,2 \pm 11,0$ & \\
\hline Amigos(as) & $62,9 \pm 13,3$ & \\
\hline Companheiro(a) & $58,5 \pm 7,9$ & \\
\hline Renda Familiar & & 0,218 \\
\hline Até $\mathrm{R} \$ 1.874,00$ & $60,9 \pm 11,0$ & \\
\hline $\mathrm{R} \$ 1.874,01$ a $\mathrm{R} \$ 3.748,00$ & $58,8 \pm 14,8$ & \\
\hline$R \$ 3.748,01$ a $R \$ 9.370,00$ & $63,0 \pm 11,1$ & \\
\hline $\mathrm{R} \$ \quad 9.370,01 \quad \mathrm{a} \quad \mathrm{R} \$$ & $66,8 \pm 14,1$ & \\
\hline $18.740,00$ & & \\
\hline $\mathrm{R} \$ 18.740,01$ ou mais & $67,3 \pm 19,7$ & \\
\hline Atividade Física & & 0,153 \\
\hline Praticante & $63,7 \pm 13,0$ & \\
\hline Não praticante & $60,3 \pm 13,2$ & \\
\hline
\end{tabular}

\section{DISCUSSÃO}

Com relação à Avaliação Global atingida pelo conjunto amostral, a média e o desvio padrão observados foram de $62,4 \pm 13,1$, com valores mínimo e máximo de 23,4 e 90,4, respectivamente.

A maioria dos estudantes que foi entrevistada para a pesquisa era do gênero feminino (61,3\%). Dados semelhantes também foram encontrados em outras pesquisas com estudantes de Odontologia de outra universidade (Universidade de São Paulo, São Paulo), onde o gênero feminino foi predominante com $72 \%$ dentre os entrevistados ${ }^{13}$.

$O$ perfil predominante dentre os entrevistados foi uma estudante do gênero feminino, com idade entre 21 e 22 anos, estudante do período integral, que apenas estuda, mora com amigos, com renda familiar entre $\mathrm{R} \$ 3.748,01$ a $\mathrm{R} \$$ 9.370,00 e praticante de atividade física. Porém, dentre os 150 entrevistados, apenas dois possuíam todas essas características e a média da qualidade de vida de ambos foi de $59,9 \pm 14,2$, ou seja, $2,5 \pm 1,1$ pontos abaixo da média geral de todos os estudantes.

Dentre os entrevistados, a classe econômica que obteve maior qualidade de vida foi a classe A (que possui renda familiar de $\mathrm{R} \$ 18.740,01$ ou mais) com $67,3 \pm 19,7$, sendo que a com pior foi a classe D (renda familiar de R\$ $1.874,01$ a $\mathrm{R} \$ 3.748,00$ ) com $58,8 \pm 14,8$, uma diferença de $8,5 \pm 4,9$, o que sugere que melhores condições financeiras refletem diretamente na qualidade de vida, além de também poder está relacionada ao fato que o curso de Odontologia ser um curso com alto gasto mensal com materiais, forçando alguns alunos a trabalharem informalmente em períodos livre para complementar a renda.

A maior parte dos estudantes entrevistados era praticante de atividade física $(61,3 \%)$. A média para os praticantes de atividade física foi de $63,7 \pm 13,0$, sendo 3,4 $\pm 0,2$ pontos a mais dos não praticantes, que obtiveram uma média de $60,3 \pm 13,2$, o que indica que atividade física eleva a qualidade de vida.

Com relação às questões levantas pelo instrumento de pesquisa, a primeira pergunta era uma autoavaliação da QV dos estudantes, a maioria (42,7\%) respondeu considerar boa a sua qualidade de vida. $\mathrm{O}$ escore de qualidade de vida geral (QVG) levantado por essa questão foi de 3,7, o que é pouco abaixo da QVG levantada em outras pesquisas com universitário ${ }^{1}$.

Para a segunda questão, que fala sobre o quão satisfeito o estudante está com a sua saúde, 39\% dos estudantes falaram estar satisfeitos, obtendo-se um escore de 3,5, que é menor que o da primeira questão, demonstrando que, para os participantes, este conceito ultrapassa uma dimensão estritamente física ou biológica o que é coerente com as definições (subjetivas e multifatoriais) sobre QV e, de modo particular, com a definição da Qualidade de Vida do Estudante (QVE) proposta por Benjamim ${ }^{14}$.

As questões 3, 4, 10, 15, 16, 17 e 18 englobavam o domínio físico, que foi o domínio com a menor média $(51,7$ $\pm 12,9)$ dentre os quatro domínios do questionário. Esse valor baixo pode estar diretamente voltado ao fato dos estudantes alegarem não estarem satisfeitos com o seu sono (questão 16 - 28\% responderam não estar satisfeitos), o que estaria correlacionado com a questão 10, que perguntava se o estudante possuía energia suficiente para o dia a dia, o que pode ser um efeito colateral da alta carga horária do curso de Odontologia.

O domínio com a maior média foi Relações Sociais $(69,3 \pm 21,2)$, que é um domínio composto por três facetas que correspondem às questões 20,21 e 22 . Entre as três questões a que obteve o menor escore foi a que perguntava "quão satisfeito você está com suas relações pessoais (amigos, parentes, conhecidos, colegas)", o que pode indicar um reflexo da situação social atual onde as pessoas estão cada vez mais individualistas e isoladas.

Constituído pelo maior número de facetas entre todos (8 ao total), o domínio ambiente é um dos mais complexos. Seu escore foi de 64,0 $\pm 17,9$, o que é acima da média geral encontrada na pesquisa. Por se tratar do ambiente físico, recursos financeiros, lar e transporte, é possível que esse valor mais elevado esteja diretamente ligado ao fato dos alunos entrevistados possuírem uma renda familiar médiaalta.

Por último, o domínio psicológico, com uma média de $64,3 \pm 12,2$, foi observado como sendo pouco mais favorável que o domínio anterior e estando também acima da média geral. Composto por seis facetas, esse fator apresenta relações diretas com o aprendizado quando questiona sobre o quanto o estudante consegue se concentrar. Além disso, estudos de Bolsoni-Silva e Guerra ${ }^{15}$ mostram que este também está ligado à aprendizagem quando questiona sobre sentimentos negativos, pois os mesmos, quando vivenciados, causam problemas à saúde mental do estudante, dificultando à assimilação do conteúdo como provado em outros estudos. 


\section{CONCLUSÃO}

Monitorar a QV da população, principalmente dos estudantes é uma necessidade premente devido às condições socioeconômicas individuais, bem como as atividades diárias curriculares e de vida. O desenvolvimento deste estudo possibilitou-nos alcançar o objetivo inicialmente proposto pela pesquisa de analisar a qualidade de vida dos estudantes de graduação e pós-graduação em Odontologia, levando-se em consideração as variáveis estudadas, para uma melhor compreensão das questões no que se refere a uma população de estudantes.

Desta forma o estudo pretendeu, de forma direta e clara, oferecer algumas contribuições para uma melhor compreensão das questões sobre qualidade de vida que se referem à população universitária de graduação e pósgraduação de Odontologia. Neste, verificaram-se indícios de que os estudantes de pós-graduação apresentavam um fator global de qualidade vida melhor que os de estudantes de graduação. Ademais, dentre os graduandos, os estudantes do integral apresentam qualidade de vida superior.

Estes resultados permitem a deteç̧ão precoce das dificuldades vivenciadas pelos estudantes de Odontologia e podem cooperar com o delineamento de estratégias que beneficiem a busca por soluções para os conflitos que incidem na qualidade de vida.

Para a melhoria da QV, algumas estratégias seriam indicadas, como a formação de grupos para a prática de atividade física, melhoria do transporte até a universidade, grupos de estudos para melhor interação entre os estudantes, bem como a disponibilidade de profissionais na universidade como psicólogos, médicos, enfermeiros e assistentes sociais. Enfatiza-se que é dever da universidade, enquanto instituição responsável pela educação dos futuros profissionais, conhecer as necessidades dos estudantes e, sobretudo, implantar estratégias que promovam o seu bem estar.

Entretanto, é válido ressaltar que embora tenha sido validado no Brasil e em outros diversos países, o WHOQOL-bref possui suas limitações, assim como outros instrumentos de avaliação de autorelato. E mesmo sendo um dos instrumentos mais utilizados, seria interessante para maior compreensão e avaliação da qualidade de vida a utilização de outros métodos, assim como a reaplicação do próprio questionário em outros momentos do semestre letivo, tanto para a graduação, quanto para a pós-graduação.

\section{REFERÊNCIAS}

1. Catunda MAP, Ruiz VM. Qualidade de vida de universitários. Pensamento Plural. 2008; 2(1): 22-31.

2. Monteiro R, Braile DM, Brandau R, Jatene FB. Qualidade de vida em foco. Rev Bras Cir Cardiovasc. 2010; 25(4):568-74.

3. Ruiz VM. Psicologia e Qualidade de Vida. In Pesquisas e Temáticas em Desenvolvimento Social emáticas em Desenvolvimento Social. UNIFAE emáticas em Desenvolvimento Social - Centro Universitário das Faculdades Associadas de Ensino-FAE (vários autores), p. 129-143. Itu (SP): Ottoni; 2005.

4. Wilheim J. O Substantivo e o Adjetivo. São Paulo: Perspectiva; 1978.

5. Limongi França AC. Treinamento e qualidade de Vida. In Boog GG. (Org.) Manual de treinamento e desenvolvimento: um guia de operações. p. 235-254. São Paulo: Makron Books; 2001.
6. The WHOQOL Group. $(\mathrm{CH})$, Division of Mental Health and Prevention of Substance Abuse. WHOQOL user manual. Geneva; 1998 Acessado em 11 de maio de $2017 . \quad$ Disponível em: http://www.who.int/mental_health/evidence/who_qol_u ser_manual_98.pdf.

7. The WHOQOL Group. The World Health Organization quality of life assessment (WHOQOL): development and general psychometric properties. 1998, Social Science and Medicine 12:1569-1585.

8. The WHOQOL Group. World Health Organization. WHOQOL: measuring quality of life. Geneva:WHO; 1997.

9. Fleck MPA, Leal OF, Louzada S, Xavier M, Chachamovich E, Vieira G et al. Desenvolvimento da versão em português do instrumento de avaliação de qualidade de vida da OMS (WHOQOL-100). Rev Bras Psiquiatr. 1999; 21(1):19-28.

10. The WHOQOL Group. WHOQOL-bref: introduction, administration, scoring and generic version of assessment. Geneva: World Health Organization, 1996

11. Sousa TF, Fonseca SA, José HPM, Nahas MV. Validade e reprodutibilidade do questionário Indicadores de Saúde e Qualidade de Vida de Acadêmicos (Isaq-A). Arq Ciênc Esporte. 2013; 1(1):21-30.

12. Fleck MPA, Louzada MX, Chachamovich E, Vieira G, Santos L, Pinzon V. Aplicação da versão em português do instrumento abreviado de avaliação da qualidade de vida "WHOQOL-bref". Rev Saúde Pública. 2000; 34(2):178-83.

13. Santos BRM, Gonzales PS, Carrer FCA, Araújo ME. Perfil e expectativas dos ingressantes da Faculdade de Odontologia da USP: uma visão integrada com as diretrizes curriculares nacionais e o sistema único de saúde. Rev ABENO. 2015; 15(1):28-37.

14. Oliveira RA, Ciampone, MHT. A universidade como espaço promotor de qualidade de vida: vivências e expressões dos alunos de enfermagem. Texto contexto enferm. 2006; 15(2):254-61.

15. Bolsoni-Silva AT, Guerra BT. O impacto da depressão para as interações sociais de universitários Estudos e Pesquisas em Psicologia. 2014; 14(2):429-52.

\section{CONFLITO DE INTERESSES}

Os autores declaram não haver conflitos de interesse.

\section{AUTOR PARA CORRESPONDÊNCIA}

João Rafael Amadeu

rafaelamadeu@foa.unesp.br

Submetido em 18/10/2017 Aceito em 17/11/2017 